\title{
Modeling of Nonlinear Isolation System Based on Bouc-Wen Differential Model
}

\section{Z. Peng and C. G. Zhou}

College of Mechatronic Engineering, North University of China, Shanxi Taiyuan, China

A feedforword neural network of multi-layer topologies for systems with hysteretic nonlinearity was constructed based on the Bouc-Wen differential model. The proposed model not only reflects the hysteresis force characteristics of the Bouc-Wen model, but can also determine the corresponding parameters. The simulation results demonstrate that the restoring force-displacement curve hysteresis loop closely represents real curves. The trained model can accurately predict the time response of the system. By comparing results obtained by the proposed model with real responses, the model was validated in the presence of noise and exhibits increased modeling precision, good generalizability and anti-interference capability.

Keywords: Bouc-Wen model, nonlinear. isolation system, modeling.

Introduction. Piezoelectric ceramic actuators such as magneto rheological damper, as well as dry friction damping steel wire rope and nonlinear delay systems exist in mechanical isolation systems, earthquake engineering, civil engineering, aerospace structural damping systems, etc. $[1,2]$. Accurate modeling is important to the analysis and response prediction of a dynamic system, and has attracted wide research attention. The Bouc-Wen model is a widely used non-linear phenomenological model which describes the smooth hysteresis behavior of the lag element according to a nonlinear differential equation $[3,4]$. The nonlinear restoring force is divided into two components: the nonlinear an hysteretic restoring force related only to the instantaneous displacement and speed of the structure, and the pure lag restoring force related to the structure of the displacement time history which can be described by a first-order nonlinear differential equation [5-7].

In the present study, the use of Bouc-Wen model is used for the topological design of the neural network layer. The corresponding relationship between network weights and the model parameters was established. A neural network model is obtained by network training, which reflects not only the hysteresis force characteristics of the Bouc-Wen model, but also the corresponding model parameters.

1. Mathematical Model of Hysteresis Nonlinear Systems. In practical engineering applications, it is necessary to establish the mathematical description of the hysteretic nonlinear force in order to analyze the hysteresis nonlinear dynamics of the system. The Bouc-Wen differential model can describe the various forms of smooth hysteresis nonlinearity [8-10]. As long as it is appropriate to change its parameters, the proposed model can describe the various types of hysteresis loops, described as follows:

$$
\begin{gathered}
R(t)=b x(t)+z(t), \\
\dot{z}=\eta \dot{x}(t)-\beta|\dot{x}(t)| z|z|^{n-1}-\gamma \dot{x}(t)|z|^{n} .
\end{gathered}
$$

Equation (2) can be rewritten as follows:

$$
\dot{Z}(t)=\eta \dot{x}(t)-\beta|\dot{x}(t)||Z(t)|^{n} \operatorname{sgn}[Z(t)]-\gamma \dot{x}(t)|Z(t)|^{n} .
$$


In Eqs. (1)-(3), $R(t)$ is the system lag restoring force, $b x(t)$ is the non-lag component, $Z(t)$ is the lag component, $b, \eta, \beta, \gamma$, and $n$ are the parameters to be identified. Among the identifiable parameters, $b, \eta, \beta$, and $\gamma$ control the shape of the hysteresis curve, while $n$ controls smoothness of the transition zone in the hysteresis curve.

2. Modeling Principles Based on the Bouc-Wen Model. The Bouc-Wen differential model reflects the relationship between the lag force and the deformation displacement. The relationship between the restoring force and deformation is determined by the five unknown parameters.

According to the relationship between the restoring force and deformation, by constructing a series of activation function, describing the differential equation by specific neural network topology structure, correspond to the network weights and model parameters. The neural network model of the system is able to obtain the lag resilience by the training of the custom network. The modeling principle is shown in Fig. 1.

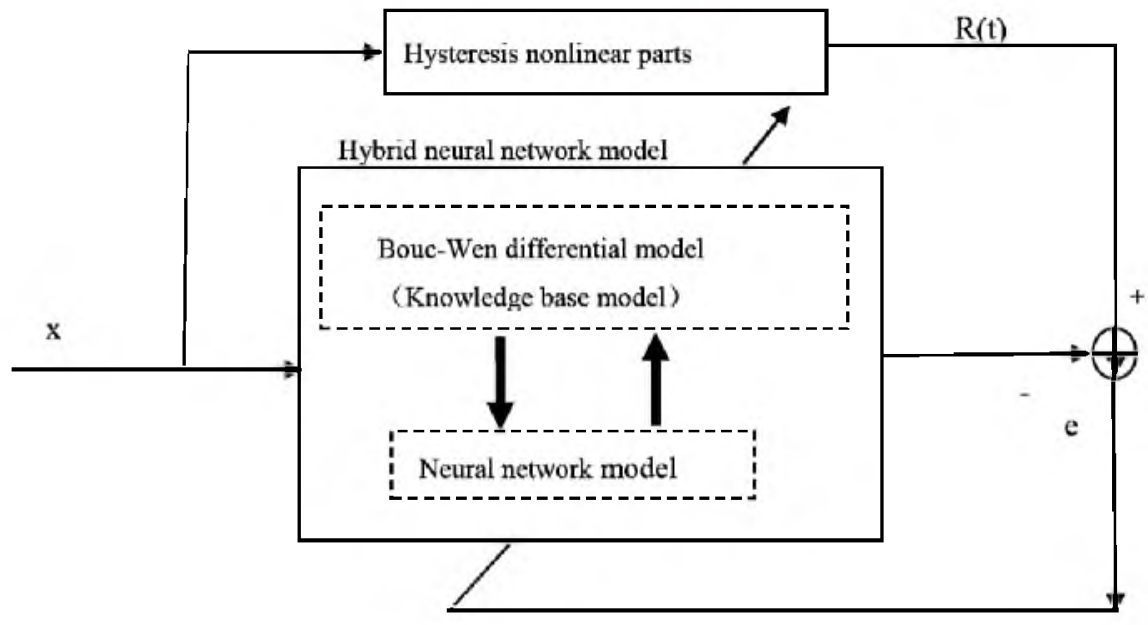

Fig. 1. Principle of hysteresis nonlinear system modeling.

3. Neural Network Topology Based on the Bouc-Wen Model. In order to construct a neural network topology, Eq. (1) must be discretized to obtain the following:

$$
\dot{R}(t)=b \dot{x}(t)+\dot{z}(t) .
$$

After the first order differential forward on Eqs. (2) and (3) can be written as follows:

$$
\begin{gathered}
\frac{r(k)-r(k-1)}{T}=\frac{b[x(k)-x(k-1)]}{T}+\dot{z}(t), \\
\dot{z}(t)=\frac{\eta[x(k)-x(k-1)]}{T}-\beta|\dot{x}(t)| z(t)|z(t)|^{n-1}-\frac{\gamma|z(t)|^{n}[x(k)-x(k-1)]}{T},
\end{gathered}
$$

where $T$ is the sampling interval, and $k$ and $k-1$ define the sampling time. Equations (5) and (6) are then combined, and the difference equation indicates the relationship between the restoring force, displacement and speed as follows:

$$
\begin{aligned}
R(k)= & R(k-1)+b[x(k)-x(k-1)]+T \eta \dot{x}(k-1)-T \beta|\dot{x}(k-1)||[R(k-1)-b x(k-1)]|^{n} \times \\
& \times \operatorname{sgn}[R(k-1)-b x(k-1)]-T \gamma \dot{x}(k-1)|[R(k-1)-b x(k-1)]|^{n} .
\end{aligned}
$$


4. Construction of Neural Network Topology. According to Eq. (7), the neural network topology shown in Fig. 2 can be constructed to achieve hysteresis nonlinearity multilayer feedforward neural network modeling between the restoring force and displacement.

As shown in Fig. 2, the model parameter information and structural information is embedded in the multilayer feedforward neural network which is integrated into the structure, and must be identified by previous knowledge of the model. In the MATLAB environment, a custom neural network is generated by the command net $=$ network, known as the init function, which initializes the network with a weight-defined initialization function to create a hybrid network, training and learning, until the requirements of training performance indicators are met.

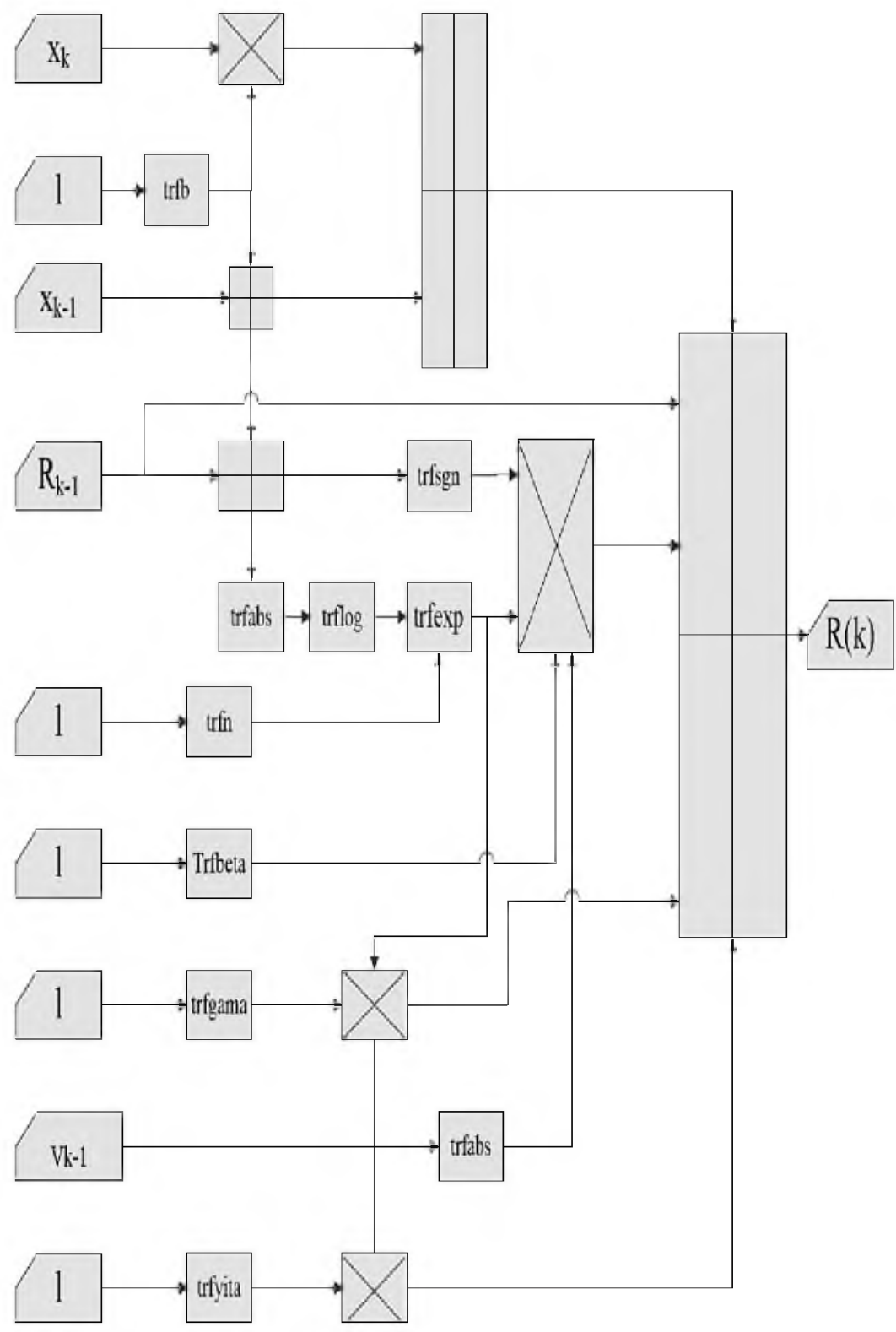

Fig. 2. Multilayer feedforward neural network modeling. 
5. Construction of Neural Network Topology. Using three groups of experimental response data, custom neural network training is achieved as shown in Fig. 2. Because the created network is static, training is achieved through the improved BP algorithm. The training result parameter values are presented in Table 1 . With the exception of parameter $\gamma$, all parameters are nearly identical to their nominal values.

$\mathrm{T}$ a b 1 e 1

Neural Network Modeling Results Based on the Bouc-Wen Model

\begin{tabular}{|c|c|c|c|c|c|}
\hline \multirow{2}{*}{ Parameter } & \multicolumn{5}{|c|}{ Parameter values training of differential model } \\
\cline { 2 - 6 } & Nominal value & No noise & $\varepsilon=5 \%$ & $\varepsilon=10 \%$ & $\varepsilon=15 \%$ \\
\hline$b$ & 0.1 & 0.1089 & 0.1317 & 0.1613 & 0.1668 \\
\hline$\eta$ & 1.0 & 0.9894 & 1.0385 & 1.0710 & 0.9468 \\
\hline$\beta$ & 0.8 & 0.9954 & 1.1689 & 1.7103 & 2.2500 \\
\hline$n$ & 1.5 & 1.4980 & 1.6111 & 1.6860 & 1.3010 \\
\hline$\gamma$ & 0.2 & 0.0060 & 0.1746 & 0.4144 & 0.3021 \\
\hline
\end{tabular}

By comparing Fig. 3, results indicate that the restoring force-displacement hysteresis loop curve and the real hysteresis loop curve are nearly identical.

As shown in Fig. 4, the contrast between the predicted steady-state response and the real system response under the three levels of motivation indicates that the training model can accurately predict the time response of the system.

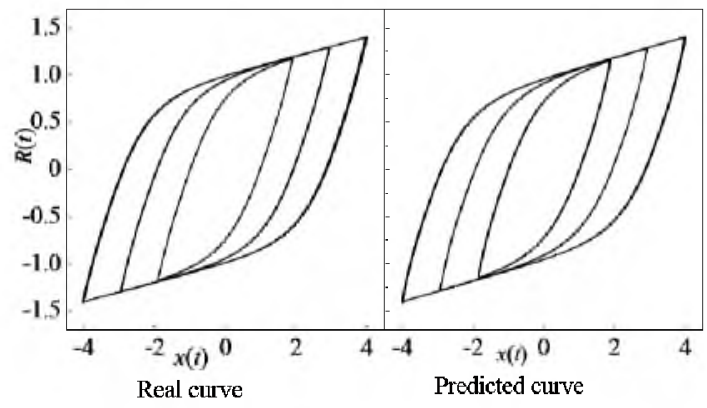

Fig. 3. Three types of real and predicted horizontal excitation resilience-displacement hysteresis curves.

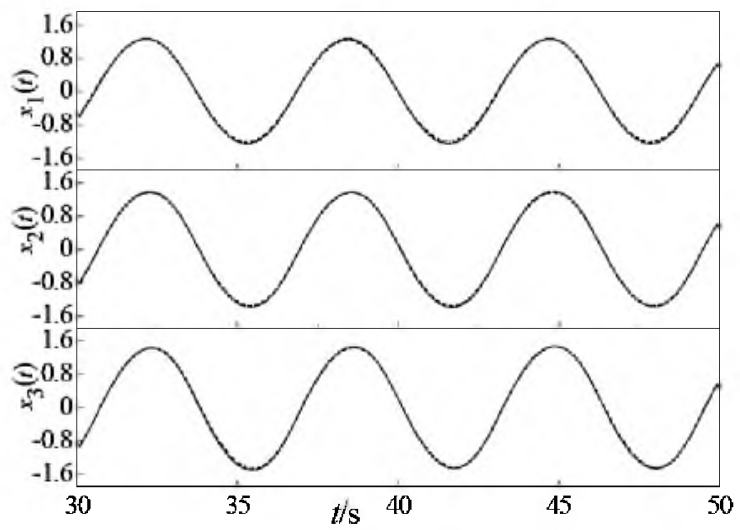

Fig. 4. Comparison of steady-state responses under the three levels of motivation (solid lines correspond to real curve and dashed lines - predicted curve). 


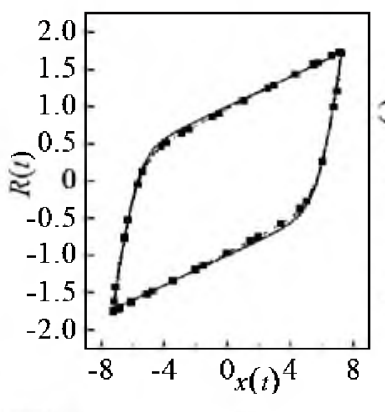

- Real curve--.-- - Prediction curve---- Predicted curve

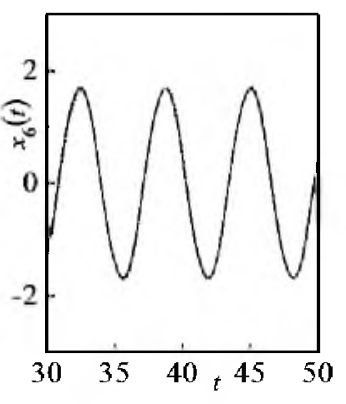

a

b

Fig. 5. Hybrid models to predict compared with real response for $\mathrm{Xg} 4=6.0$ : (a) restoring force; (b) acceleration.

In order to test the training ability of the hybrid network model, the predicted response is calculated and compared to the real response, as shown in Fig. 5. The hybride network model is still able to accurately predict the system response and the hysteresis curve, and exhibits good generalizability.

6. Model Performance in the Presence of Noise. It is assumed that the restoring force data representing the three levels of motivation is polluted by noise, expressed as follows:

$$
R_{j}(t)=R_{j}(t)+\varepsilon r_{j} R_{j 0},
$$

where $r_{j}$ is the normal distribution with zero mean unit variance random signal sequence, $R_{j 0}$ is the magnitude of the restoring force $j$, and $\varepsilon$ is the noise level.

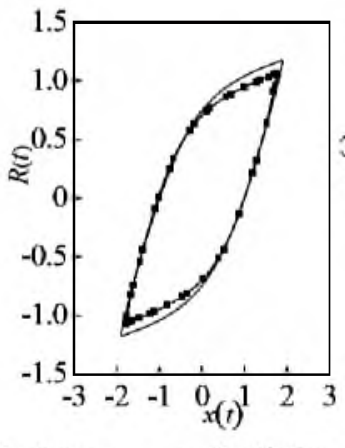

- Real curve ...... Prediction curve -- Predicted curve

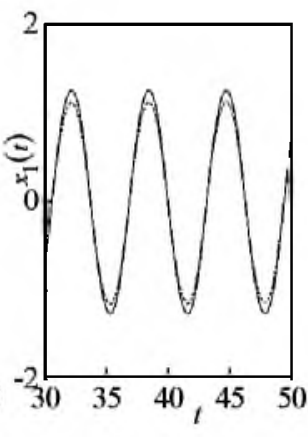

a

b

Fig. 6. Hybrid models to predict compared with real response for $\varepsilon=5 \%$ : (a) restoring force; (b) acceleration.

The hybrid network is separately trained in the use of data where $\varepsilon$ is equal to 5 , 10 , and $15 \%$. The training parameters are shown in Table 1 . When the noise level is equal to $5 \%$, the training parameters exhibit some deviation. However, the simulation model is still able to accurately predict the response of the system with hysteresis characteristics as shown in Fig. 6. Additionally, the results depicted in Table 1 also indicate that the value of the error parameter during training gradually increases with noise level. Thus, the influence of noise can be reduced by increasing the training sample data. 


\section{Conclusions}

1. In the present study, a Bouc-Wen differential model of delayed nonlinear systems was presented, a multilayer feedforward neural network model of neural network topology was constructed and the proposed model was trained with experimental response data.

2. Simulation results indicate that the obtained restoring force-displacement hysteresis loop curves and the real hysteresis loop curves were nearly identical, demonstrating that the trained model can accurately predict the time response of the system.

3. Results indicate that the model exhibits good generalizability based on comparison with real response data.

1. Y. Tan, Z. He, and J. T. Gao, "Isolation analysis of low-frequency vibration induced by high-speed railway," J South China Univ. Technol. (Natur. Sci. Edit.), 39, No. 6, 132-136, 154 (2011).

2. Y. K. Wen, "Method for random vibration of hysteretic systems," J. Eng. Mech. Div., 102, No. 2, 249-263 (1976).

3. A. Carrella, M. J. Brennan, I. Kovacic, and T. P. Waters, "On the force transmissibility of a vibration isolator with quasi-zero-stiffness," J. Sound Vib., 322. Nos. 4-5, 707-717 (2009).

4. Z. Ye, A. Sadeghian, and B. Wu B, "Mechanical fault diagnostics for induction motor with variable speed drives using Adaptive Neuro-fuzzy Inference System," Electr. Pow. Syst. Res., 76, Nos. 9-10, 742-752 (2006).

5. M. Reza Mashinchi and A. Selamat, "An improvement on genetic-based learning method for fuzzy artificial neural networks," Appl. Soft Comput., 9, No. 4, 1208-1216 (2009).

6. I. Kovacic, M. J. Brennan, and T. P. Waters, "A study of a nonlinear vibration isolator with a quasi-zero stiffness characteristic," J. Sound Vib., 315, No. 3, 700-711 (2008).

7. P. Ciarlini and U. Maniscalco, "Wavelets and Elman Neural Networks for monitoring environmental variables," J. Comput. Appl. Math., 221, No. 2, 302-309 (2008).

8. Z. Z. Wang, "The study of complex system modeling and simulation on evolution base," J. Syst. Simul. (S1004-731X), 15, No. 7, 905-909 (2003).

9. S. H. Li and S. P. Yang, "Research status of hysteretic nonlinear models," J. Dyn. Control, 4, No. 1, 8-15 (2006).

10. J. S. Lu, R. Fang, and X. J. Lan, "Hot research areas of simulation technique in the country - Review of Journal of System Simulation in recent years," J Syst. Simul. (S1004-731X), 16, No. 9, 1910-1913 (2004). 